\title{
Kommentare
}

\section{Christopher Pollmann* Zwischen Conseil d'Etat und Auswendiglernen}

Keine »einheitliche«, sondern vielgestaltige Juristenausbildung in Frankreich

Wenn in Deutschland eine Berufsgruppe besonders viel Macht und Einfluss ausübt, dann sind es wohl die Juristen. Dementsprechend lässt sich die universitäre Befassung mit Recht als Aneignung von Herrschaftswissen verstehen, und die juristische Ausbildung ist der einzige Zugang zu dieser Welt von Macht und nicht selten auch Reichtum. Ganz anders in Frankreich. Zwar ist auch >[d] as [französische] Recht ein Herrschaftsdiskurs $<{ }^{1}{ }^{1}$ doch wird er nicht unbedingt von vollausgebildeten Juristen gehalten. Das mag die deutschen »Einheitsjuristen« verblüffen, weil sie selbst ein relatives Monopol in der Rechtsanwendung geniessen.

Deshalb soll der folgende Beitrag die juristische Ausbildung in Frankreich sowohl beschreiben als auch kritisch analysieren. Zunächst werden Organisation und Inhalte des universitären Jurastudiums vorgestellt (I). Im Unterschied zu Deutschland gibt es im Anschluss daran für wesentliche juristische Berufe spezialisierte Ausbildungen (II). Übergreifend sollen zuletzt Ziele und Methoden der juristischen Ausbildung erörtert werden (III). Wollte man insoweit die Lage in Frankreich mit Deutschland vergleichen und pointiert charakterisieren, so ließe sich sagen: Um Jurist zu werden, muss man in einer - durch die sog. Grandes écoles (Großen Schulen) - stark hierarchisierten Ausbildungslandschaft Essays schreiben, die mittels eines Plan einheitlich zu gliedern sind.

Sowohl die Rechtsordnung als auch das Ausbildungssystem in Frankreich unterscheiden sich erheblich von den deutschen Verhältnissen. Um die französische Juristenausbildung verständlich zu machen, sind deshalb drei einleitende Schritte erforderlich. Sie werden uns mit der Stellung des Rechts und der Juristen im Nachbarland, der Struktur der Hochschulausbildung und der Lehre des Rechts in verschiedenen Studiengängen vertraut machen.

\section{Die Rolle des Rechts und der Juristen in Frankreich}

Die Juristen sind die Kleriker der Marktwirtschaft, die vom Gesetz und der Gerichtsentscheidung organisiert wird. Das Recht als moderne Mythologie² oder

\footnotetext{
* Ich danke meinem Kollegen und Freund Hugues Rabault für seine hilfreichen Hinweise.

I Vgl. Danièle Loschak, "Le droit, discours de pouvoir«, Festschrift Léo Hamon, Economica: Paris I982, S. 429-444 (meine Übers.).

$2 \mathrm{Vgl}$. Jacques Lenoble/François Ost, Droit, mythe et raison. Essai sur la dérive mytho-logique de la rationalité juridique, Publications des Facultés universitaires St. Louis: Brüssel I980.
} 
als Fetisch ${ }^{3}$ bedeutet für diese Gesellschaftsordnung soviel wie die Theologie für den

Feudalismus. ${ }^{4}$ Doch einige Besonderheiten der französischen Geschichte und der Pariser Zentralismus haben die Entwicklung und die Bedeutung des Rechts in Frankreich bis heute gehemmt, insbesondere auf dem Gebiet des öffentlichen Rechts.

Die Französische Revolution setzte einerseits das Werk des monarchischen Ancien régime fort, indem sie die Zentralisierung auf die Spitze trieb und zunehmend einen in Sprache und öffentlichem Bewusstsein homogenen, immer schärfer nach außen abgegrenzten Nationalstaat schuf.' (Das bedeutet allerdings keineswegs, dass damit die Gesellschaft auch materiell vereinheitlicht worden wäre, wie der sog. Kulturalismus unterstellt; auch das französische Volk ist »nur « eine simaginäre Gemeinschaft.$\left.^{6}\right)$ Die Überhöhung des Politischen begünstigte einen öffentlichen Voluntarismus, ${ }^{7}$ der namentlich die idealistische Verneinung materieller Interessen umfasst, z. B. in der - in Frankreich noch stärker als in Deutschland präsenten - Ideologie der dem gesamten Volke und nicht bloß ihren Wählern oder ihrem Wahlkreis verpflichteten Abgeordneten in der Nationalversammlung, während das Mehrheitswahlrecht offiziös ihre lokale Verankerung voraussetzt und fördert. Andererseits - und gerade auch wegen dieses Voluntarismus' - hat die Revolution die bereits unter der Monarchie begonnene Verrechtlichung in mehrfacher Hinsicht gestört.

Die Entwicklung der Rechtsnormen wurde, bildlich gesprochen, an der Spitze wie an den Wurzeln behindert. Die Nationalversammlung sah sich als Verkörperung des Volonté générale (des allgemeinen Willens) Jean-Jacques Rousseaus, und das von ihr verabschiedete Gesetz galt als Ausdruck des >Gesellschaftsvertrages.$^{8}$ Das jeweils jüngste Gesetz als aktuellste Äußerung des Volkswillens war von daher heilig. Zwar wurden während der Revolutionsjahre I789-95 neben der Erklärung der Menschenund Bürgerrechte vom 26.8. I789 drei Verfassungen verabschiedet (I79I, I793 und I795), doch war damit nicht gemeint, die zukünftige Gesetzestätigkeit einzuengen. Für die Nationalversammlung war nicht vorstellbar, dass ein Text der Vergangenheit ihr die Hände binden könnte. Auf diese Weise kam die Bedeutung der Verfassungsnormen als »Haupt « des Rechtssystems praktisch nicht zur Geltung; sie konnten und können teilweise noch heute - nicht nur durch Gesetze, sondern in bestimmten Konstellationen sogar durch Verordnungen der Regierung oder der Verwaltung gebrochen werden. 9 Paradoxerweise ließen die Souveränität des Gesetzgebers und die sich daraus speisende oder diese gar ablösende Autorität der Regierung einen

3 Vgl. Pierre Legendre, Jouir du pouvoir. Traité de la bureaucratie patriote, Editions de Minuit: Paris 1976, namentlich S. I $33 \mathrm{ff}$.

4 Vgl. meinen Besprechungsaufsatz »Zur Objektivierung von Herrschaft durch Recht «, über: Hugues Rabault, L'interprétation des normes: l'objectivité de la méthode herméneutique, L'Harmattan: Paris 1997, in: Archiv für Rechts- und Sozialphilosophie 1999, S. 282-288. Vgl. auch D. Loschak (Fn. I).

5 Vgl. mit zahlreichen Nachweisen C. Pollmann, "La frontière - horizon indépassable de l'humanité ou pouvoir objectivé? «, Revue du droit public et de la science politique 1999, S. 48 I-499 (vor allem Abschnitt II, S. 486 ff.); englische Kurzfassung in: Xpovıка [Chroniken], Band 9, Sept. 1996, Athen/Komotini I997, S. $47-53$.

6 Benedict Anderson, Imagined communities. Reflections on the origin and the rise of nationalism, Verso: London/New York, überarb. u. erw. Neuaufl. i991 (Die Erfindung der Nation. Zur Karriere eines folgenreichen Konzepts, erw. Neuausgabe, Frankfurt/M. 1996); Jean-François Bayart, L'illusion identitaire, Fayard: Paris 1996.

7 Vgl. aus unterschiedlichen Blickwinkeln u. a. Ladan Boroumand, La guerre des principes. Les assemblées révolutionnaires face aux droits de l'homme et à la sowveraineté de la nation mai 1789 -juillet I794, Vorwort Mona Ozouf, Ed. de l'EHESS: Paris I999; François Furet, Penser la Révolution française, Gallimard: Paris 1985 .

8 Jean-Jacques Rousseau, Du contrat social ou principes $d u$ droit politique (1762), Flammarion: Paris 1992 (Vom Gesellschaftsvertrag oder Grundlagen des politischen Rechts, Frankfurt/M. 2000).

9 Gemäß der im Rückzug befindlichen Theorie vom >loi-écran`: Das Gesetz als Schirm zwischen Verfassung und Richter verhindert, dass letzterer eine Verordnung auf ihre Verfassungsmäßigkeit überprüfen darf, wenn dadurch indirekt auch ein der Verordnung zugrundeliegendes Gesetz kontrolliert würde, ständige 
konsequenten Vorrang theoretisch höherrangiger Normen nicht zu und behinderten so die Verrechtlichung. ${ }^{\text {10 }}$

Was die Wurzeln der Rechtsordnung angeht, so betrachtet der Zentralismus die Gebietskörperschaften - zunächst handelte es sich um die Gemeinden und Départements, inzwischen ergänzt durch die Regionen - bis heute als bloße Verwaltungseinheiten ohne politische Regelungsmacht. Die Kompetenz, eigene Rechtsnormen zu verabschieden, wird ihnen überwiegend abgesprochen. Die von ihren demokratisch gewählten Vertretern beschlossenen Regeln sind nicht nur dem nationalen Gesetz, sondern auch der Verordnungsgewalt der Regierung und ihrer Minister untergeordnet. ${ }^{\text {II }}$

Auch die Entwicklung der Justiz verlief nur zögerlich. Da die Richter der vorrevolutionären Zeit als Verteidiger feudaler Privilegien galten, brachten die Revolutionäre ihnen großes Misstrauen entgegen. Zwecks Trennung zwischen - legislativer und administrativer - Normsetzung und gerichtlicher Kontrolle untersagt ein noch heute gültiges Gesetz vom I6. und 24. 8. I790 den Richtern, in ihren Urteilen zukünftige Entscheidungen $\mathrm{zu}$ präjudizieren, also allgemeine Regeln aufzustellen, oder die Anwendung von Gesetzen und teilweise sogar von Verordnungen auszusetzen (Artikel ro und I 2), womit die Kontrolle ihrer Verfassungsmäßigkeit nicht in Betracht kommen kann. ${ }^{22}$ Ferner ist den Richtern - in tendenziell allerdings sinkendem Umfang - verboten, der Verwaltung Weisungen zu erteilen, und sei es auch nur zu dem Zwecke, die sich aus einem Urteil ergebenden Verpflichtungen zu erfüllen (Artikel I 3 ). ${ }^{13}$

Die Justiz ist aus diesen Gründen deutlich geringer ausdifferenziert und die Zahl der Richter wie auch der Juristen überhaupt erheblich niedriger als etwa in Deutschland und vor allem den angelsächsischen Ländern. ${ }^{14}$ Es gibt lediglich die allgemeine Gerichtsbarkeit mit zusätzlichen erstinstanzlichen Arbeitsgerichten und Sozialgerichten, die Verwaltungsgerichtsbarkeit sowie den erst 1958 geschaffenen und zunächst einflusslosen Conseil constitutionnel (wörtlich: Verfassungsrat). Allerdings hat die Verabschiedung des Code civil im Jahre I 804 all diesen Tendenzen auf dem Gebiet des Privatrechts entgegengewirkt. Behindert wurde von daher vor allem die Rechtsprechung im öffentlichen Recht und die Herausbildung des Verfassungsrechts, insbesondere der Grundrechte. Letztere sind in der derzeit gültigen Verfassung von 1958 lediglich global durch Verweis auf die o. g. Erklärung von i 789 erwähnt, und das auch nur in der Präambel. Die Verfassungsväter waren sich nämlich durchaus

Rechtsprechung, z. B. Conseil d'Etat, Urteil v. 28. 7. 99, Rs. Griesmar, Recueil (Entsch.-samml.), S. 248. Vgl. René Chapus, Droit administratif général, Bd. I, I3. Aufl., Montchrestien: Paris I999, Nr. 5 I f., S. 3 I f.

ı Vgl. Marie-Jeanne Redor, De l'Etat légal à l'Etat de droit. L'évolution des conceptions de la doctrine publiciste française I 879-1914, Economica \& Presses universitaires d'Aix-Marseille I992. Sie zeigt aber auch (S. 225 f.), dass der Conseil d'Etat in Reaktion auf die Souveränität des Gesetzgebers die Gesetzmäßigkeit von Verordnungen und Verwaltungsakten zunehmend überprüfte.

I I Art. 72 VI Verfassung, L. 2I3 I-6, L. 3I32-I u. L. 4I42-I Code général des collectivités territoriales; vgl. Bertrand Faure, Le pouvoir réglementaire des collectivités locales, L.G.D.J.: Paris I998, S. 2 I6-23 I; JeanMarie Becet, Les institutions administratives, 3. Aufl., Economica: Paris I992, S. 21 9-22 3; Jean-Marie Auby, »Décentralisation et sources du droit«, L'actualité juridique - droit administratif, Sonderausgabe April I992, S. 30-34.

I 2 Zu diesem Absatz vgl. Bernard Chantebout, Droit constitutionnel, 20. Aufl., A. Colin: Paris 2003, S. I 2 I. Der Bedarf an - auch - gerichtlicher Normsetzung hat den Conseil d'Etat jedoch veranlasst, ein praktisch höchst aktiver Richter zu sein: Das französische Verwaltungsrecht ist weitgehend sein Werk.

I 3 Conseil d'Etat v. 28. 2. I996, Rs. Fauqueux, Recueil S. 52; vgl. ausführlich zur komplexen Rechtslage René Chapus, Droit du contentieux administratif, 6. Aufl., Montchrestien: Paris 1998, Nr. 1086-1 107, S. 7998 I 5 .

I4 In Frankreich sind 2004 ca. 47.500 (»Voll«)Juristen tätig (vgl. Anne Crignon, Les métiers du droit, L'Etudiant: Paris 2004, S. 8), was zwar eine starke Zunahme gegenüber 1992 bedeutet (ca. 32.000 lt. Dominique \& Michèle Frémy, Quid 2004, R. Laffont: Paris 2003, S. 809), aber bescheiden bleibt im Vergleich zu Deutschland (ca. I90.000 Juristen lt. Financial Times Deutschland v. I6. 10. 2002) und insbesondere den USA (s. Frémy, ibid.). 
bewusst, dass die effektive Anerkennung von Grundrechten Verrechtlichung wie auch

Individualisierung bedeutet, ${ }^{\text {is }}$ Entwicklungen also, denen sie ablehnend gegenüberstanden. Bis zu einer Entscheidung des Conseil constitutionnel im Jahre I97 I zur Vereinsfreiheit ${ }^{16}$ hatten die Menschen- und Bürgerrechte deshalb mangels Rechtsprechung kaum normative Wirkung.

Auch die schriftliche Dokumentation von Rechtsnormen, Rechtsprechung und Dogmatik lässt im Vergleich zu wünschen übrig. Das Ineinandergreifen von kodifizierten, d.h. in Gesetzes- und Verordnungsbüchern zusammengefassten, und nichtkodifizierten Vorschriften ${ }^{17}$ samt ihrer nachträglichen Änderungen sowie die Zersplitterung der veröffentlichten Gründe einer Gerichtsentscheidung auf den meist extrem kurzen Text der Entscheidung (im wesentlichen die sog. Considérants) und die Erwägungen des Commissaire du gouvernement (bei der Verwaltungsgerichtsbarkeit) bzw. des Generalanwalts (vor Zivil- und Strafgerichten) machen die Rechtslage häufig unübersichtlich. Die Zitierweise von Vorschriften ist uneinheitlich und umständlich, Gesetzeskommentare existieren kaum, und Gesetzessammlungen wie Lehrbücher enthalten meist nur sehr kurze Sachregister; Urteilsregister fehlen regelmäßig.

Insgesamt ist die Verrechtlichung in Frankreich also weniger ausgeprägt als in vielen anderen kapitalistischen Ländern. Gesellschaftliche Konflikte sind dementsprechend in stärkerem Umfang politisiert. Die andernorts zu beobachtende Rolle des Rechts als Herrschaftstechnik wird in Frankreich teilweise von Politik- und Ingenieurwissenschaften eingenommen. Nicht Juristen, sondern Absolventen der Grandes écoles, ${ }^{18}$ nämlich vor allem der Ingenieur- und Verwaltungshochschulen, an ihrer Spitze die Ecole polytechnique, die Ecole nationale d'administration (ENA) und das Institut d'études politique in Paris (»Science po«) nehmen in Politik, Verwaltung, Justiz und großteils auch der Privatwirtschaft die Spitzenpositionen ein. ${ }^{19}$ Z. B. sind die Richter am Conseil d'Etat (wörtlich: Staatsrat), dem höchsten Verwaltungsgericht, aber nach wie vor auch Beratungsorgan der Regierung, keine vollausgebildeten Juristen, sondern sog. ENArques!

Genauer gesagt, konzentriert sich die juristische Ausbildung der Verwaltungselite auf das Verwaltungsrecht, das innerhalb des öffentlichen Rechts die dominierende Stellung einnimmt. Das französische Verwaltungsrecht seinerseits stellt im wesentlichen die Verrechtlichung von Hierarchie und einseitiger Entscheidungsmacht des Staates dar. ${ }^{20}$ Historisch lässt es sich als formalisiertes Rückzugsgebiet der von der Französischen Revolution und der Demokratisierung erschütterten monarchischen Gewalt

Is Vgl. C. Pollmann, „Die Berufung auf Menschenrechte zum Zweck ihrer Überwindung. Menschenrechte, kapitalistischer Staat und soziale Bewegungen«, Widersprüche Nr.46, April I993, S. 4I-52; ders., »Individualisme, double mesure et pathos moral. Une critique des droits de l'homme illustrée par des propos d'écrivains et de philosophes ", Revue de la coopération transfrontalière Nr. 29, Mai 200I, S. 22-29 (25-27 m. w. N., namentl. auf Karl Marx, »Zur Judenfrage«, in: I. Fetscher (Hrsg.), Marx-Engels Studienausgabe, Bd. I, Frankfurt/Main i 966, S. 3 I-60).

I6 Entscheidung 44 DC vom 16.7. 1971, Recueil S. 29. Mit Kommentar und w. N. abgedruckt bei Louis Favoreu/Loïc Philip, Les grandes décisions du Conseil constitutionnel, 8. Aufl., Dalloz: Paris I995, S. 244$26 \mathrm{I}$.

I7 Vgl. beispielhaft zum Umweltrecht Philippe Billet, "La lettre et le néant: De l'inconsistance de la codification dans le domaine des pollutions, des nuisances et des risques «, Droit de l'environnement Nr. 85 , Jan 2001 , S. 2 I-25.

I 8 Zum (Un)Wesen der Grandes écoles, vgl. Pierre Bourdieu, La noblesse d'Etat: Grandes écoles et esprit de corps, Editions de Minuit: Paris I989 (Der Staatsadel, Konstanz 2004); Gilles Larzuch, L'Exception française. Le modèle des grandes écoles à l'épreuve de la mondialisation, Presses universitaires de Rennes I 999.

I9 Seit I 870 und erneut ab 1945 entgleitet die Ausbildung der Verwaltungselite den juristischen Fakultäten, deren Unterricht als zu rechtstechnisch und traditionell gilt, vgl. Jacqueline Gatti-Montain, Le système d'enseignement $d u$ droit en France, Presses universitaires de Lyon 1987, S. I $28-139$.

$20 \mathrm{Vgl}$. Valérie Larrosa, Recherches sur la notion de hiérarchie en droit administratif français, Diss. Universität Toulouse I, I998. 
analysieren. Die prunkvolle Selbstdarstellungsherrschaft der Monarchie mutierte so zur bürokratisch-diskreten Macht des kapitalistischen Nationalstaates. ${ }^{21}$ Insofern ist die Verrechtlichung in Frankreich nicht nur geringer entfaltet als in anderen liberalkapitalistischen Ländern, sondern auch stärker hierarchisch und autoritär geprägt. Trotz dieser kritischen Kurzanalyse des französischen Rechts- und Politiksystems soll Verrechtlichung hier keineswegs als zwangsläufig positives Phänomen verstanden werden. Dazu sei hier in Kürze lediglich gesagt, dass rechtliche Regulierung neben zivilisierenden Wirkungen auch schwer durchschaubare Herrschaft, Vereinzelung und Entfremdung mit sich bringt. ${ }^{22}$ In den USA geht die Verrechtlichung im Übrigen mittlerweile so weit, dass den Jurastudenten dort mit der Doctrine (oder Theory) of efficient breach of contract ${ }^{23}$ beigebracht wird, Kosten und Nutzen eines Vertragsbruches kühl gegeneinander abzuwägen. Das dadurch steigende Risiko von Vertragsbrüchen zwingt die Vertragspartner wie auch die Gesamtgesellschaft zu zusätzlichem Aufwand für juristische Beratung und Verteidigung. Das Recht wird so zur Last!

\section{Das Hochschulstudium im allgemeinen}

Wichtigstes Merkmal des französischen Ausbildungssystems ist wohl die starke Hierarchisierung sowohl in fachlicher als auch in geographischer Hinsicht. ${ }^{24}$ Grundlegender noch genießen handwerkliche, manuelle und praktische Tätigkeiten und Ausbildungen (soweit es letztere überhaupt gibt) allgemein einen schlechten Ruf; die entsprechenden Verrichtungen sind häufig von bescheidener Qualität. Im uns interessierenden Bereich sieht die Rangordnung vereinfacht so aus: An der Spitze steht die $E N A$, dicht gefolgt von Science $p o^{25}$ und - weiter unten-den anderen Instituts d'études politiques (z. B. in Strasbourg, Grenoble, Bordeaux). Erst dann treten die juristischen Fakultäten in Erscheinung. Das Schlusslicht bildet der gleichfalls an Universitäten angebotene Studiengang Administration économique et sociale (AES). Ähnliche Hierarchien gibt es in den anderen Sozial- und Kulturwissenschaften (an der Spitze stehen die Ecoles normales supérieures im Pariser Raum, vor allem jene in der rue d'Ulm), den Wirtschaftswissenschaften (geführt von einigen, teilweise privaten Managementschulen) sowie den Natur- und Ingenieurwissenschaften (die Ecole polytechnique und die Ecole centrale des arts et manufactures führen eine Vielzahl von Grandes écoles an, die in einer offiziell anerkannten Rangfolge stehen). Richard Descoings, Leiter von Sciences po, fasst die Situation weitblickend und auch erstaunlich (selbst)kritisch so

2I Michel Miaille, Vorlesung im Verwaltungsrecht an der Universität Montpellier I. Etymologisch ist Bürokratie die Macht der Büros, d. h. eine nicht auf Waffen, Prunk, Heiligtümer oder Charisma, sondern auf Schriftverkehr und Akten gestützte Herrschaft.

22 Vgl. z. B. David Kairys (Hrsg.), The Politics of Law. A Progressive Critique, Basic Books: New York 3. Aufl. 1998; Duncan Kennedy, A Critique of Adjudication (fin de siècle), Harvard University Press: Cambridge/ Mass. I997; D. Loschak (Fn. I) sowie die in Fn. Is genannten Schriften.

23 Begründet von Robert Birmingham, "Breach of Contract, Damage Measures and Economic Efficiency «, 24 Rutgers Law Review 1970, S. 273 ff. Für eine aktuelle Erörterung, vgl. Melvin A. Eisenberg, »Remedial Theory In Contract Law: Compensation; the Theory of Efficient Breach; Cover; Actual and Virtual Specific Performance; and Disgorgement « (2004), www.law.berkeley.edu/institutes/law_econ/ workingpapers/PDFpapers/Eisenberg2_so4.pdf.

$24 \mathrm{Vgl}$. dazu umfassend und kritisch Marie-Laure de Léotard, Le dressage des élites. De la maternelle aux grandes écoles, un parcours pour initiés, Plon: Paris $200 \mathrm{I}$.

${ }_{25} \mathrm{Zu}$ diesen beiden Schulen vgl. Alain Garrigou, »Die Kaderschmiede der französischen Nation: »Sciencespo« - die gewöhnliche Funktion einer ungewöhnlichen Institution«, Le Monde diplomatique (mit der Tageszeitung) v. I 2.3. I999, S. 20 f., www.monde-diplomatique.de/pm/I 999/o3/I 2/a00 I I.text.name,askrOmEqe.n,7; ders., »Comment Sciences Po et l'ENA deviennent des `business schools«, Le Monde diplomatique (frz. Ausgabe), Nov. 2000, S. 24 f. 
zusammen: >Für die Grandes écoles gibt es eine Zukunft nur insoweit, als die Universitäten keine Zukunft haben [...], als es keine Revolution der Universitäten gibt, eine Revolution, die ich mir wünscher. ${ }^{26}$

Vor diesem Hintergrund wählen französische Abiturienten ihren Studiengang nicht in erster Linie aus Neigung, sondern aufgrund des juristisch sanktionierten oder auch nur inoffiziellen Arbeitsmarktwertes der Fachrichtung und des Studienortes. Das hat zur Folge, dass zahlreiche Einrichtungen der weiterführenden Bildung eine Mehrheit mit sich selbst unzufriedener, von mangelndem Selbstvertrauen geplagter Studenten beherbergen. Wer beispielsweise Jura studiert, tut dies häufig, weil es ihr oder ihm nicht gelungen ist, an einer der Politik- und Verwaltungshochschulen oder einer anderen Grande école aufgenommen zu werden; $A E S$ bleibt dann für diejenigen übrig, die sich auch zum Jurastudium nicht in der Lage sehen. (Dabei erscheint mir dieser Studiengang aufgrund seiner interdisziplinären Anlage weitaus vielversprechender als Jura.) Während sich in anderen Ländern in allen, auch den exotischsten Fächern selbst an den unbedeutendsten Universitäten brillante Studenten einfinden, ist mir in Metz deshalb in Jura wie insbesondere in AES bislang noch kein einziger Student - unter mittlerweile knapp 3000 - als herausragend begabt und motiviert aufgefallen. Das allerdings liegt auch an der geographischen Hierarchisierung der Universitäten, bei der Metz schlecht abschneidet: Wenn sie nicht sowieso eine Grande école im Auge haben, gehen aufgeweckte Abiturienten aus Metz zum Studium lieber nach Nancy (65 km südlich) oder Strasbourg ( $60 \mathrm{~km}$ östlich).

Die große Zahl passiver und eingeschüchterter Studenten ist letztlich auf die pädagogische Situation an der französischen Schule zurückzuführen. ${ }^{27}$ Gefordert werden dort vor allem formale Fähigkeiten (wie sauberes Schreiben und das Erstellen einer Gliederung, des sog. Plan) und insbesondere erschöpfendes Mitschreiben und Auswendiglernen zwecks Wiedergabe bei Prüfungen. Nachdenken, Argumentieren und Diskutieren, Lebendigkeit und Problembewältigung spielen demgegenüber eine untergeordnete Rolle. ${ }^{28}$ Das dürfte auch der Grund für die geringe Verbreitung von Fremdsprachenkenntnissen wie auch musikalischen Fertigkeiten (die sich eben nur durch Aktivität erwerben lassen) in Frankreich sein. Vor diesem Hintergrund ist verständlich, dass Formalismus und Frontalunterricht die französische Bildungs- und Forschungslandschaft bis in ihre Spitzen charakterisiert, z. B. am Collège de France und der sozialwissenschaftlichen Elitehochschule Ecole des hautes études en sciences sociales (EHESS) wie auch in den Concours (den anonymen Zugangsprüfungen des öffentlichen Dienstes und der Grandes écoles) für die Besetzung der höchsten Positionen, insbesondere der Agrégation.

Das Studienjahr beginnt im September/Oktober, wird nach dem ersten Semester unterbrochen durch schriftliche Examen und endet im Mai/Juni mit einer zweiten Folge schriftlicher und mündlicher Prüfungen. Die dabei durchgefallenen Studenten können an zwei Wiederholungsexamen, meist im September, teilnehmen. Die Notenskala reicht von $\circ$ bis 20; ab der Note 10 ist eine Prüfung bestanden. Nach jedem Studienjahr ermöglichen auf ein Hundertstel genau ermittelte Abschlussnoten die Einordnung der Absolventen in eine klare Rangfolge. Diese Note und vor allem der Platz in der Rangordnung sind für die weitere Laufbahn - Zugang zu anderen

26 Richard Descoings, zit. bei Justine Ducharne, »Ya-t-il encore des grandes écoles en France«, Le Figaro v. 5.5 .2004 (meine Übers.).

27 Vgl. Jean-Michel Djian, Le triomphe de l'ordre. La pensée tuée par l'école, Flammarion: Paris 2000; J.-P. Le Goff, La barbarie donce. La modernisation avengle des entreprises et de l'école, La Découverte I999; und für Philosophie, Fach des letzten Gymnasialschuljahres, Patrick Rayou, La >Dissert de philor. Sociologie d'une épreuve scolaire, Presses universitaires de Rennes 2002.

28 Vgl. Jean-Yves Fournier, "L'intelligence à l'école«, Sciences humaines Nr. I 16, Mai 200I, S. 34-37. 
Bildungsgängen, Stipendien, Zuschüsse für Veröffentlichungen, Anstellung im öffentlichen Dienst u. a. - stets von immenser Bedeutung.

Die Fakultätsverwaltungen sowie der Lehrkörper verwenden erhebliche, wenn nicht die meiste Energie auf die Anwesenheitskontrolle in den Übungen, die Reglementierung, praktische Vorbereitung und Überwachung der Prüfungen, die Entanonymisierung und Archivierung der Klausuren, die von den Studenten nach Korrektur nur unter Aufsicht - und in der Praxis deshalb nur selten - eingesehen werden können, usw.

Positiv ist anzumerken, dass zumindest die universitären Studiengänge bislang bereits nach mindestens zwei Jahren mit dem Diplôme d'études universitaires général (DEUG) abgeschlossen werden können, nach drei Jahren mit der Licence und nach vier Jahren mit der Maîtrise. ${ }^{29}$ Darauf folgen einjährige Vertiefungsprogramme, im wesentlichen das Diplôme d'études approfondies $(D E A)$, das der Vorbereitung auf eine Doktorarbeit dienen soll, und das berufsvorbereitende Diplôme d'études supérieures spécialisées (DESS). Studenten, die nicht bis zu den entsprechenden Abschlussexamen vorstossen, haben somit immerhin ein Diplom in der Hand, während in Deutschland vor dem Magister oder der Staatsprüfung nach mindestens vier- bis fünfjährigem Studium kaum anerkannte Abschlüsse existieren.

Zur räumlichen Mobilität der Studenten ist zu sagen, dass die große Mehrheit unter ihnen im Heimatort oder an der nächstgelegenen Hochschule studieren. Erst in einem fortgeschrittenen Stadium, meist nach der Licence oder der Maîtrise, wechseln manche Studenten die Hochschule. Der Hauptgrund ist jedoch auch dann nicht der Wunsch nach Veränderung und neuer Umgebung, sondern die angestrebte Spezialisierung, die an der bisherigen Hochschule nicht immer angeboten wird. Der gleichen Logik folgend rekrutieren sich Hochschullehrer vorwiegend unter den vor Ort tätigen Studenten und Doktoranden bzw. Doktoren. Nicht selten haben Professoren ihr gesamtes Studium an nur einer Universität absolviert, nämlich dort, wo sie auch beruflich tätig sind!

Das ist umso erstaunlicher, als der voluntaristische Zentralismus bei zahlreichen Berufsgruppen des öffentlichen Dienstes eine extreme Mobilität erzwingt und meint, damit örtliche Bindungen und Interessen aus der Welt schaffen zu können. (Die meisten Lehrer, Professoren, Richter, Polizisten u. v. a. m. werden entweder von der jeweiligen Ministerialverwaltung kreuz und quer durch Frankreich geschickt. Oder sie müssen hoffen, im jeweiligen Concours weit oben in der Rangfolge zu landen, um eine Stelle daheim oder an einem Ort ihrer Wahl besetzen zu können. Dabei haben die von einer Stellenbesetzung betroffenen Behörden in der Regel keinerlei Möglichkeit, an der Auswahl von Bewerbern mitzuwirken.) Aber vielleicht ist gerade diese Zwangsmobilität einer freiwilligen Beweglichkeit in Raum und Beruf abträglich. Um in eine Grande école aufgenommen zu werden, ist es nötig, neben dem Abitur noch eine mehr oder minder strenge, anonyme Eingangsprüfung zu bestehen. Das

29 Vgl. den Arrêté v. 9. 4. 1997, Journal officiel - Lois et décrets v. I 5. 4. 1997, S. 5679 ff., inzwischen mehrfach geändert. (Alle in diesem Beitrag genannten Gesetze und Rechtsverordnungen lassen sich über www. legifrance.gouv.fr auffinden.) Im Rahmen der Reform >Licence - Master - Doctorat $<$ soll allerdings die Organisation der Studiengänge und Universitäten grundlegend verändert werden. Die drei genannten Diplome würden nach jeweils mindestens drei, fünf bzw. acht Studienjahren vergeben; DEUG und Mầtrise sind nicht mehr vorgesehen. Ziel ist neben einer europaweiten Harmonisierung vor allem die Rationalisierung des Universitätsbetriebes zwecks Einsparung finanzieller Mittel, namentlich durch Zusammenlegung kleinerer Einheiten. Die dadurch hervorgerufenen Proteste wie auch die allgemein als verworren und unverständlich eingeschätzte Architektur des angestrebten Systems lassen es zweifelhaft erscheinen, ob erstens die Reform überhaupt durchgeführt wird und ob zweitens die überkommenen Strukturen nicht wie häufig gleichwohl - lediglich neu gewandet - beibehalten werden. Aus diesen Gründen soll die Erörterung des französischen Hochschulwesens hier nicht - womöglich unnötig - durch die Einbeziehung der Reformpläne belastet werden. 
Studium an einer Universität ist hingegen ohne weitere Prüfung möglich; auch ein Numerus clausus (Erfordernis einer bestimmten Abiturnote angesichts eines begrenzten Studienplatzangebotes) ist selten, nämlich hauptsächlich in Medizin und Pharmazie. Dafür sind Studienabbruch und Durchfallquoten bei Examen an Universitäten wesentlich höher als in den Grandes écoles, die man, einmal aufgenommen, kaum ohne Abschluss wieder verlässt. Zur Vorbereitung auf deren Eingangsprüfungen ist es regelmäßig erforderlich, im Anschluss an das Abitur während mindestens zwei Jahren sog. Classes préparatoires zu absolvieren. Es handelt sich meist um regelrechte "Paukkurse«, die in den Gymnasien oder bereits an der jeweiligen Grande école stattfinden. Das Studium an den Grandes écoles ist insofern paradox, als die meiste Lernarbeit nicht dort, sondern in diesen Vorbereitungsklassen stattfindet, während es im Interesse der psychosozialen Entwicklung junger Menschen sinnvoll wäre, zwischen 16 und 20 Jahren möglichst viel freie Zeit zu haben. ${ }^{\circ}$ Schon die Gymnasien stehen in einer offiziösen Rangordnung, die von der Erfolgsquote ihrer Schüler bei den verschiedenen Grandes écoles und deren Vorbereitungsklassen abhängt. Trotz zentralisierter und anonymer Prüfungen entspricht dieser Hierarchie im Übrigen auch eine soziale Schichtung: Je größer das Prestige eines Studienganges, je kleiner der Anteil an Arbeiterkindern (etwa $5 \%$ an den Grandes écoles); ein Kind mit leitendem Angestellten als Vater hat $50 \mathrm{mal}$ mehr Aussichten, auf die Ecole polytechnique zu gehen, als ein Arbeiterkind. ${ }^{31}$ Überdies haben sich dessen Chancen auf eine Spitzenausbildung in den letzten 30 Jahren halbiert.

Während an den Universitäten Massenvorlesungen und schlechte materielle Ausstattung überwiegen, sind die Studienbedingungen an den Grandes écoles meist erheblich besser, insbesondere Unterricht in Klassenstärke und gut gefüllte Bibliotheken. An den bedeutendsten Grandes écoles haben die Studenten, obwohl erst (mindestens) I9 oder 20 Jahre alt, bereits Beamtenstatus, beziehen ein Gehalt und haben nach erfolgreichem Studienabschluss Anspruch auf eine Stelle im öffentlichen Dienst. Die Dienststellen sind inoffiziell ihrerseits hierarchisiert: Unter den jährlich etwa ı०० französischen - neben ca. 40 ausländischen - Absolventen der ENA z. B. können die besten sich ihre Arbeitsposten aussuchen und werden meist zu prestigeträchtigen Einrichtungen wie der Inspection des finances (ministerielle Haushaltskontrolle), dem Conseil d'Etat oder dem Cour des comptes (Rechnungshof) gehen. Die letzten müssen mit den übrigbleibenden Stellen Vorlieb nehmen; sie werden sich dann häufig auf irgendwelchen Provinzpräfekturen wiederfinden. (Die Präfektur ist die Vertretung des Staates im Département; sie steht neben dem vom Volk gewählten Conseil général.) Aber immerhin, mit (mindestens) 22 oder 23 Jahren schon die Stelle eines Unterpräfekten bekleiden zu dürfen, ist ohne Zweifel ein beruflicher Schnellstart...

\section{Die Lebre des Rechts in verschiedenen Ausbildungsgängen}

Wie schon angedeutet, wird Recht nicht nur im Studiengang Jura (Droit), sondern auch in anderen Ausbildungsgängen an Universitäten wie Grandes écoles gelehrt. An den verschiedenen Politik- und Verwaltungshochschulen stellen die juristischen die wichtigsten Unterrichtsmaterien dar. Das spiegelt die große Bedeutung wider, die dem öffentlichen Recht in den Concours eingeräumt wird. Schließlich sind auch in AES mindestens ein Drittel der Unterrichtsstunden dem Recht gewidmet. Gleichwohl 
gehört Recht mit Medizin zu den beiden einzigen Fächern, die in einem vollen Studiengang nur an Universitäten, nicht aber an einer Grande école angeboten werden.

Die hiermit zu Tage tretende Zersplitterung der juristischen Lehre auf verschiedene Ausbildungsgänge ist wahrscheinlich ein zusätzlicher Grund für die vergleichsweise unauffällige Stellung von Juristen in der französischen Gesellschaft. ${ }^{32}$ Es gibt eben rechtliche Kenntnisse und Fertigkeiten auch bei vielen anderen Hochschulabsolventen, ohne dass den Universitätsjuristen irgendwelche Privilegien oder gar ein Monopol zugebilligt wären. Mit anderen Worten: Frankreich kennt keine »einheitliche Juristenausbildung « und folglich auch keinen Einheitsjuristen. Zwar haben auch hier Rechtsanwälte, auf dem Verfahrenswege definiert mittels einer durchaus vereinheitlichten Ausbildung und Prüfungsordnung (s. u.), ein relatives Monopol in der Rechtsberatung und Prozessvertretung. ${ }^{33}$ Doch im Unterschied zu Deutschland strahlt diese Geschlossenheit nicht auf andere juristische Berufe aus: Um in der deutschen Terminologie zu sprechen, sind die Zulassung zum Rechtsanwalt, die Befähigung zum Richteramt und die Befähigung zum höheren Verwaltungsdienst in keiner Weise aneinander gekoppelt. ${ }^{34}$

Diese Vielfalt im Auge, wollen wir uns nun schwerpunktmäßig mit dem Studiengang Jura (I) und der darauf folgenden spezialisierten Ausbildung für wesentliche juristische Berufe (II) befassen. Abschließend sollen die Ziele und Arbeitsmethoden der juristischen Ausbildung beleuchtet werden (III).

\section{Organisation und Inhalte des universitären Jurastudiums ${ }^{35}$}

Wie auch in Deutschland ist im Prinzip das Abitur (Baccalauréat) erforderlich, um zu studieren. ${ }^{36}$ Für Personen ohne Abitur gibt es hiervon zwei beschwerliche Ausnahmen mit bescheidenen Erfolgsaussichten. Das innerhalb von zwei Jahren, in Ausnahmefällen auch während eines Jahres erwerbbare Diplôme d'accès aux études universitaires (Hochschulzugangsdiplom) berechtigt dazu, ein beliebiges Studium aufzunehmen. Sodann ist die Capacité en droit zu nennen. Es handelt sich dabei um eine juristische Grundausbildung im Rahmen mindestens zweijähriger Abendveranstaltungen. Wurde sie trotz hoher Abbruch- und Durchfallquote (über 90\%) erfolgreich abgeschlossen, können die meist schon berufstätigen Absolventen ein juristisches - mit besonderer Genehmigung auch ein anderes - Studium aufnehmen.

Innerhalb der Universitäten wird Jura an juristischen Fakultäten gelehrt. Da der Begriff Faculté wie auch in Deutschland den »Muff unter den Talaren « symbolisierte, wurden sie Ende 1968 in Unités d'enseignement et de recherche (UER, Lehr- und Forschungseinheiten) oder Unités de formation et de recherche (UFR, Ausbildungsund Forschungseinheiten) umbenannt. ${ }^{37}$ Diese umständliche und uneinheitliche Bezeichnung hat sich aber nur teilweise durchgesetzt. Viele Fachbereiche - wie man in Deutschland nun auch sagen würde - bezeichnen sich weiterhin oder erneut als

32 Diese beiden, im Vergleich zu Deuschland u. a. Ländern auffälligen Aspekte (also Zersplitterung und unauffällige soziale Stellung) werden nicht hinreichend berücksichtigt in der kaum rechtsvergleichend angelegten Studie von J. Gatti-Montain (Fn. I9).

33 Gesetz Nr. 7I-I I 30 v. 3 I. I 2. I97 I, Journal officiel - Lois et Décrets v. 5. I. I972, S. I I Iff.

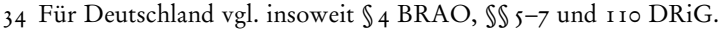

35 Zahlreiche - von mir teilweise aktualisierte - Einzelheiten verdanke ich dem Frankreich-Bericht von JeanClaude Masclet und Nathalie Rebby für die Tagung >La formation juridique dans l'Europe de demain< der EG-Kommission und der Universität Metz, 27.-29. I0. I994. Vgl. ferner den Arrêté vom 30. 4. I997, Journal officiel - Lois et décrets v. 4. 5. 1997, S. $6766 \mathrm{ff}$., inzwischen mehrfach geändert.

36 Art. L. 6r 2-3 I Code de l'éducation. Hierzu u. zum folgenden vgl. Art. 9 des o. g. Arrêté v. 9. 4. I 997 (Fn. 29).

37 Art. 3 f. des Gesetzes Nr. 68-978 v. I 2. I I. I968, Journal officiel-Lois et Décrets v. I 3. I I. I 968 , S. I0579ff.

(Das Gesetz selbst ist inzwischen aufgehoben worden.) Vgl. auch Art. L. 7I 3-I Nr. 2 Code de l'éducation. 
Fakultäten. Dieses Scheitern eines auch sprachlichen Voluntarismus ist symptomatisch für die unablässigen, aber meist hilf- und wirkungslosen Versuche der Pariser Ministerialbürokratie, das Hochschulwesen (wie auch andere Bereiche des öffentlichen Dienstes) zu reformieren.

Die ersten drei Studienjahre (DEUG und Licence) sind Frankreich-weit ähnlich, während mit der Maîtrise die universitätsspezifische Spezialisierung einsetzt. Das zweijährige Grundstudium bis zum DEUG darf vier, mit Einzelfallerlaubnis maximal fünf Jahre dauern. Die Erlangung der Licence und der Maîtrise kann beliebig oft versucht werden.

Gleichwohl sind Abbruch- und Durchfallquoten hoch. Etwa $80 \%$ der Studienanfänger in Jura bleiben bis zum Abschluss des ersten Jahres dabei; von diesen gelingt lediglich $30 \%$ der Übergang ins zweite Studienjahr. Berücksichtigen wir die Wiederholbarkeit des ersten Jahres, so gelangen ungefähr $30-40 \%$ der Studienanfänger ins zweite Jahr, I 5-20\% erreichen das DEUG und zwischen 10 und I $5 \%$ die Licence.

Um diese geringe Erfolgsquote zu verbessern, wurde das Grundstudium I998 »semestrialisiert«, d. h. in zwei Semester mit jeweiligen Prüfungen und Wiederholungsprüfungen (bei einjährigen Veranstaltungen also nunmehr insgesamt vier) aufgeteilt. Die einmal erfolgreich abgeschlossenen Fächer oder Fächergruppen (Module) brauchen dann bei Jahrgangswiederholung nicht noch einmal belegt zu werden. Sie können auch dann als erworben gelten, wenn die Studenten nach dem ersten Semester in ein verwandtes Studium wechseln, also z. B. von Jura nach AES, Economie et gestion (Volks- und Betriebswirtschaft) oder in die Sozial- und Humanwissenschaften. Das erste Semester soll also neuerdings auch eine Orientierungsphase für diejenigen sein, die in ihrer Studienwahl noch unsicher sind. Es bleibt fraglich, ob diese mehr oder minder »künstliche « Hebung der Erfolgsquote die Lage der Universitäten und ihrer Studenten grundlegend bessern wird. Denn die beiden schwerwiegendsten Probleme kommen so gar nicht in den Blick, nämlich die schulmäßige Konditionierung junger Menschen auf Passivität und die Hierarchisierung höherer Bildung durch die Grandes écoles.

Das Unterrichtsvolumen bis zum DEUG beträgt mindestens roo० Zeitstunden (in Frankreich ist die »akademische Viertelstunde« cum tempore unbekannt, aber bei mehreren zusammenhängenden Stunden sind die Pausen eingerechnet). Bei etwa 25 Vorlesungswochen pro Jahr ergibt das ein Wochenpensum von mindestens 20 Stunden. An juristischen Fächern werden in dieser Zeit hauptsächlich Zivilrecht, Verfassungsrecht, Verwaltungsrecht und Strafrecht gelehrt. Teilweise sollen diese bereits sozialwissenschaftliche Elemente enthalten; z. B. heißt die Vorlesung des Verfassungsrechts offiziell Droit constitutionnel et institutions politiques. Aber die hier geforderte politische Institutionenkunde gebärdet sich häufig dann doch sehr juristisch. Wir werden darauf noch zurückkommen. Hinzu treten verschiedene mehr oder weniger explizit sozialwissenschaftliche Lehrveranstaltungen, die aber - soweit es nicht um Wirtschaft geht - regelmäßig auch von Juristen durchgeführt werden, und zwar vor allem: Internationale Beziehungen und Organisationen, Europäische Institutionen, Rechts- und Institutionengeschichte, Öffentliches Finanzwesen, Politologie und Wirtschaftswissenschaften.

Die vorgenannten Studienfächer müssen wenigstens $60 \%$ des gesamten Unterrichtsvolumens umfassen, also mindestens 600 Stunden. Für die verbleibenden $40 \%$ können die Universitäten bzw. Jurafakultäten Veranstaltungen eigener Wahl anbieten, und zwar entweder zur Vertiefung der o. g. Grundlagenfächer oder zur Verbreiterung in Richtung auf andere Disziplinen. An der Universität Metz etwa werden bis zum DEUG zusätzlich noch Politische Ökonomie, Wirtschafts- und Sozialgeschichte, Geschichte der politischen Ideen und Politiksoziologie gelehrt. 
Obligatorisch sind weiterhin eine Fremdsprache und Informatik. Doch ihr Stundenumfang ist nicht festgelegt und kann deshalb sehr niedrig ausfallen. In Metz dauert der Fremdsprachenunterricht während der ersten beiden Jahre immerhin 8I Zeitstunden.

Für das weitere Studienjahr bis zur Licence mit mindestens 500 Unterrichtsstunden nehmen folgende juristische Fächer wenigstens 300 Stunden ein: Verwaltungsrecht, Grundfreiheiten (Libertés publiques, die bezeichnenderweise nicht zum Verfassungsrecht gehören), Zivilrecht, Recht und Steuern der Unternehmen, Sozialrecht, Völkerrecht, Europarecht, Rechtsgeschichte und Geschichte der Institutionen bzw. der politischen Ideen, Steuerrecht und Gerichtsverfassungsrecht (soweit diese beiden Fächer noch nicht bis zum DEUG gelehrt worden sind). Hinzu kommt wiederum eine Fremdsprache (in Metz werden hier 26,5 Stunden angeboten). Daneben treten teilweise noch Geschichte und Wirtschaft.

Das vierte Studienjahr bis zur Maîtrise umfasst erneut mindestens 500 Unterrichtsstunden, von denen wenigstens 350 Stunden universitäre Lehrveranstaltungen darstellen. Der Rest dient einem Praktikum mit Bericht oder der Abfassung einer Forschungs- und Studienarbeit (Hausarbeit). Neben der allgemeinen juristischen Maîtrise gibt es noch neun weitere, spezialisierte Maîtrises, und zwar: Privatrecht, Öffentliches Recht, Unternehmensrecht, Zivil- und Strafjustizlaufbahn und Strafrecht, Sozialrecht, Notarrecht, Europarecht, Völkerrecht, Rechtsvergleichung. Zwei dieser Diplome können auch gemeinsam erworben werden. I 50 Stunden an universitären Lehrveranstaltungen müssen der jeweiligen Spezialisierung gewidmet sein.

An manchen Fakultäten gibt es als Diplom zudem noch einen Master oder Magister. Diese Abschlüsse krönen ein zweijähriges, anspruchsvolles Zusatzstudium, das Studenten mit der Licence offensteht. Doch die zahlenmäßig stark begrenzten Studienplätze werden über einen strengen Numerus clausus verteilt; das bewirkt, dass unter den Bewerbern viele bereits eine Maîtrise besitzen. Unter dem Einfluss der Studentenaustauschprogramme (Erasmus) bieten einige Fakultäten auch DEUG, Licences und Mâttrises an, die binationale Studiengänge abschließen.

Wie auch in anderen Ländern ist die juristische Ausbildung stark durch die Abgrenzung zwischen öffentlichem und Privatrecht beeinflusst. Rechtsgeschichte bildet eine eigenständige Disziplin gleichen Ranges, während Strafrecht zum Privatrecht gehört. Auch andere stark vom Staat geformte Fächer gelten als privatrechtlich, insbesondere Sozialrecht, Gerichtsverfassung und Prozessrecht. Gleichwohl dürfte - im Unterschied etwa zu Deutschland - das öffentliche Recht die gegenüber dem Privatrecht dominante Disziplin sein. Das liegt wahrscheinlich an der zentralen Rolle des Staates und an dem schon erläuterten Prestige der Verwaltungselite: Die Juristen mit dem weitaus meisten Ansehen sind eben, von den neun berufenen Verfassungsrichtern abgesehen, nicht Hochschullehrer oder gar Anwälte, sondern Conseillers d'Etat!

Das Unterrichtspensum der auf Lebenszeit angestellten Hochschullehrer (Maîtres de conférences und Professeurs agrégés) beträgt übrigens I 8 Zeitstunden im Jahr, also gut 5 Wochenstunden; die Vergütung ist etwa halb so hoch wie in Deutschland. Hochschullehrer verfügen meist weder über wissenschaftliche Mitarbeiter noch über Schreibkräfte. Das bedeutet auch, dass Doktoranden und Assistenten kaum der in Deutschland verbreiteten Ausbeutung durch Professoren unterliegen. Die Berufung auf Lebenszeit erfolgt in der Regel mehr oder weniger schnell nach Abschluss der Dissertation auf eine Stelle als Maître de conférences; die Agrégation (ein langwieriger Concours in den drei juristischen Disziplinen, den Wirtschaftswissenschaften und Politologie) oder die Habilitation (in den anderen Fächern) erlauben dann die Vergabe einer Professorenstelle. 
Die meisten Studenten streben einen Abschluss ihres Jurastudiums mit der Maîtrise an. Dieses Diplom ist nicht nur für einen $D E A$ mit eventuell anschließender Dissertation, sondern auch für die spezialisierten Ausbildungen zu den verschiedenen juristischen Berufen erforderlich oder jedenfalls ratsam. Mit dem Universitätsstudium ist die juristische Ausbildung für viele nämlich noch nicht beendet. Die Tätigkeit als Richter, Rechtsanwalt, Notar, Gerichtsvollzieher, Konkursverwalter u. a. bedarf des erfolgreichen Abschlusses einer Zusatzausbildung samt vergüteter Stage (Praxisstation ähnlich wie im deutschen Referendariat). Nur die juristische Tätigkeit in Unternehmen ist insoweit nicht geregelt.

Angehende Zivil- und Strafrichter sowie Staatsanwälte finden sich für ein gutes Jahr an der Ecole nationale de la magistrature in Bordeaux ein, wo sie bereits einen Beamtenstatus und Gehalt haben. Der praxisbezogene Unterricht wird durch Gerichtspraktika ergänzt. Theoretisch kann sich erstaunlicherweise jeder Inhaber einer - auch nichtjuristischen - Maîtrise für einen Ausbildungsplatz an der Richterhochschule bewerben. ${ }^{38}$ Aber die strenge Eingangsprüfung - auf eine Stelle kommen I 6 Bewerber - fragt natürlich vor allem nach Rechtskenntnissen und -fertigkeiten. Praktisch kommen deshalb die aufgenommenen Bewerber fast alle von juristischen Fakultäten oder von Science po, wo eine erfolgversprechende Prüfungsvorbereitung angeboten wird. Außerdem können sich Absolventen der ENA sowie Hochschullehrer mit juristischer Qualifikation für eine Erprobungszeit von maximal fünf Jahren und dann endgültig auf eine Stelle als Richter oder Staatsanwalt abordnen lassen. ${ }^{39}$

Auch Verwaltungsrichter bedürfen keines juristischen Studiums. Eine beliebige Licence oder eine mindestens vierjährige Berufserfahrung reichen aus, um an einem der vier Concours teilzunehmen. $4^{\circ}$ Die drei Concours der $E N A^{4 \mathrm{I}}$ und die erfolgreich absolvierte Ausbildung an derselben erlauben den Prüfungsbesten den Eintritt in den Conseil d'Etat und den weniger brillanten Absolventen die Einstellung bei einem Tribunal administratif (Verwaltungsgericht), von denen es über Frankreich verteilt 37 gibt, oder bei einem der acht Cours administratives d'appel (Oberverwaltungsgerichte). Wer es nicht bis zur ENA gebracht hat, kann derzeit auch noch über einen vierten, ausschließlich der unteren Verwaltungsgerichtsbarkeit dienenden Concours Verwaltungsrichter werden. ${ }^{22}$ Eine fünfte Zugangsmöglichkeit steht Beamten mit mindestens ıоjährigem Dienstalter sowie Zivil-, Strafrichtern und Staatsanwälten offen und besteht ausnahmsweise nicht aus einem Concours, sondern einer Einzelfallprüfung. ${ }^{43}$ Richter an einem Tribunal administratif können nach 4 jähriger Tätigkeit zu einem Cour administrative d'appel aufsteigen.

Die Ausbildung zum Rechtsanwalt erfolgt an 33 Centres régionaux de formation professionnelle des avocats, die dem örtlichen Barreau (Anwaltskammer) oder dem jeweiligen Cour d'appel (vergleichbar mit einem Oberlandesgericht) unterstehen und seit einigen Jahren vom Conseil national des barreaux koordiniert werden. 25 bis $30 \%$

38 Für die Einzelheiten vgl. Art. I 5-I7 der Ordonnance Nr. 58-I 270 portant loi organique v. 22. I 2. I958 (Journal officiel - Lois et Décrets v. 23. I 2. 58 , S. I I 5 I ff.) mit späteren Änderungen.

39 Art. $4 \mathrm{I}$ bis $4 \mathrm{I}-9$ Statut de la magistrature, eingefügt durch Loi organique Nr. 92-1 89 v. 25. 2. 1992, Journal officiel-Lois et Décrets v. 29. 2.92.

40 Hierzu und zum laufenden Absatz vgl. Art. L. 233-I bis L. 233-6 Code de justice administrative.

4I Der sog. externe Concours gilt für Inhaber einer Licence, die jünger als 28 Jahre sind. Der interne Concours richtet sich an höchstens 35 jährige Beamte mit mindestens ${ }_{4}$ Dienstjahren. Schließlich können sich auch Personen aus der Privatwirtschaft sowie politische Mandatsträger bewerben, wenn sie jünger als $4 \circ$ Jahre sind und 8 Jahre Berufserfahrung haben (3. Concours). Vgl. Art. I ff. Décret Nr. 82-819 v. 27.9. 1982 (Journal officiel - Lois et Décrets v. 28.9. 82, S. 2874 ff.) mit späteren Änderungen.

42 Dieser Concourssteht mindestens 2 sjährigen Inhaberneiner Licence,Zivil-,Strafrichternund Staatsanwälten sowie Beamten mit mindestens 7 Dienstjahren offen, vgl. Art. L. 233-6 Code de justice administrative.

43 Vgl. Art. L. 233-3, R. 233-4 u. R. 233-5 Code de justice administrative. 
der Bewerber, die eine juristische Maîtrise oder ein gleichwertiges Diplom haben müssen, ${ }^{44}$ gelingt die Aufnahmeprüfung. Die Ausbildung dauert mindestens drei Jahre und findet in zwei Etappen statt. Das erste Jahr umfasst Lehrveranstaltungen und zwei Kurzpraktika von insgesamt drei Monaten; es endet mit dem Anwaltsexamen für das Certificat d'aptitude à la profession d'avocat. Dann folgen zwei Jahre praktischer Tätigkeit bei einem Rechtsanwalt, unterbrochen von vertiefenden Lehrveranstaltungen. Nach diesen dreiJahren kann der junge Anwalt entweder als solcher beruflich tätig sein oder eine Spezialisierung anstreben. Zu diesem Zweck muss er entsprechende Forschungsarbeit und Veröffentlichungen erbringen oder eine vierjährige Schulung im gewählten Gebiet absolvieren und ein abschließendes Examen bestehen.

Die Ausbildung zum Notar ist mit insgesamt vier Varianten stark ausdifferenziert. Die ersten beiden Möglichkeiten erfordern eine juristische Maîtrise. Ihr folgen zwei jeweils einjährige Spezialisierungen: entweder ein weiteres universitäres Studienjahr zur Erlangung eines DESS im Notarrecht, an das sich Prüfungen zwecks Erwerbs des Diplôme supérieur du notariat anschließen, oder die Vorbereitung auf das gleichwertige Diplôme d'aptitude aux fonctions de notaire in einem Ausbildungszentrum für Notare. Eine anschließende zweijährige Stage in einem Notariat und begleitende Seminare verschaffen dann in beiden Fällen die nötige Erfahrung. Eine dritte Möglichkeit ist berufsintern: Die Inhaber eines DEUG in Jura können Notargehilfe werden und nach neun Jahren beruflicher Praxis das Diplôme d'aptitude erwerben. Viertens können schließlich auch erfahrene Juristen Notar werden.

Die Ausbildung zum Gerichtsvollzieher (Huissier) erfordert eine Licence in Jura und erfolgt durch eine zweijährige Stage in einer Kanzlei und praxisorientierte Lehrveranstaltungen an der nationalen Kammer der Gerichtsvollzieher, die auch die Abschlussprüfung abnimmt. Die Tätigkeiten als Gerichtsvollzieher und als Notar genießen keine Niederlassungsfreiheit, denn es handelt sich nicht nur um »freie« Berufe, sondern auch um öffentliche Ämter. Die Zahl der Kanzleien ist reglementiert; um sich niederzulassen, ist es nötig, ein Amt bzw. eine Kanzlei zu kaufen.

Konkursverwalter brauchen eine juristische oder betriebswirtschaftliche Maîtrise sowie eine dreijährige Stage, die von einer mündlichen Prüfung - als reiner Formalität - abgeschlossen wird. Im Unterschied zu den beiden vorgenannten Berufen herrscht hier Niederlassungsfreiheit.

Die Aufnahme in die öffentliche Verwaltung kann hier nicht im einzelnen dargestellt werden. Die schon erwähnten Concours sind äußerst zahlreich. Gleichwohl gibt es keine nur Juristen offenstehende Concours.

\section{Ziele und Methoden der juristischen Ausbildung}

Was sind die Ziele der juristischen Ausbildung in Frankreich? Gesetze und Verordnungen geben insoweit nicht viel her. ${ }^{45}$ Zwei miteinander in Verbindung stehende Pole lassen sich ausmachen. Zum einen muss die juristische Ausbildung praxistaugliche Juristen hervorbringen; wenn wir hier einmal vorläufig eine idealistische - d. h. die offizielle - Sicht einnehmen, sollte es deren Aufgabe sein, gesellschaftliche Konflikte mit Hilfe des Rechts zu lösen. Zum anderen kann aber auch erstrebenswert sein, das Recht zu erforschen, zu verstehen und zu erklären.

44 Hierzu und zum vorangegangenen Satz vgl. Décret Nr. 9I-I I 97 v. 27. I I. I 99I mit späteren Änderungen, Art. 5 I- 55 nennen die Zugangsbedingungen (Journal officiel-Lois et Décrets v. 28. I I. 9I, S. I 5502 ff.). Für die weiteren juristischen Berufe wird hier auf Nachweise verzichtet.

45 Vgl. Art. 2 u. s des o. g. Arrêté v. 30.4. 1997 (Fn. 35). Zu Zielen und Aufgaben der höheren Bildung allgemein vgl. Art. L. I23-I bis L. I23-9 Code de l'éducation. 
Die erste Orientierung führt zur Vermittlung von Rechtstechnik, die zweite zur

Entwicklung von Rechtswissenschaft. In den anderen Studiengängen und Fakultäten gibt es einen ähnlichen Dualismus zwischen berufsorientiertem und theoretischem Lernen. Aber während z. B. in Politologie, Psychologie und Linguistik der wissenschaftliche Ansatz und die von ihm verlangte Interdisziplinarität mehr oder minder breiten Raum einnehmen, existiert er an den juristischen Fakultäten nur in ganz bescheidenem Masse, wenn überhaupt. ${ }^{6}{ }^{6}$ Das gilt sowohl für Frankreich als auch für Deutschland und manche andere Länder.

Der Grund dafür dürfte in der eingangs vermuteten Rolle des Rechts als Rahmen und Mittel moderner Herrschaft liegen. Anzunehmen ist, dass Recht gerade deshalb und insoweit in einer Gesellschaft funktioniert, als die ihm zugrundeliegenden Mechanismen nicht verstanden werden, auch nicht von den Juristen selbst. Diesem Erfordernis wird u. a. dadurch Rechnung getragen, dass die juristische Ausbildung eine Erziehung zur Hierarchie durchführt, d.h. hierarchisches Denken und Arbeiten begünstigt. ${ }^{47}$ Die Einfügung der Juristen in Hierarchien ist in Frankreich aufgrund des staatsfixierten öffentlichen Rechts möglicherweise besonders ausgeprägt. So betrachtet, behindert Rechtswissenschaft die Rechtstechnik geradezu. Das könnte erklären, warum sich Juristen, wenn sie denn forschend tätig sind, so viel beharrlicher als in anderen Bereichen interdisziplinären Methoden verweigern..$^{8}$

Die übliche Befassung etwa mit einem Gerichtsurteil beschränkt sich darauf, den Tenor und die tragenden Gründe mehr oder minder textimmanent auszulegen, mit anderen Entscheidungen in Verbindung zu setzen und - in Auseinandersetzung mit gleichartigen Analysen - in eine übergreifende Systematik einzuordnen. Überlegungen zu den beiden Grundfragen wissenschaftlicher Betätigung, nämlich >wie?< und 'warum?<, also zum Wechselspiel zwischen dem einzelnen Urteil und kollektiven wie individuellen Interessen, Handlungsformen und -zwängen, zum Gewicht wirtschaftlicher Zweckmäßigkeit, sprachlicher Präjudizierung und psychologischer Beeinflussung fehlen meist völlig. Das rein beschreibende Vorgehen der herrschenden Dogmatik (in Frankreich Doctrine) drückt sich zugespitzt in Titeln und Überschriften aus, die nach Kräften von Erklärungsversuchen und - zwangsläufig persönlichen - Wertungen bereinigt sind. Das spiegelt die Idealvorstellung vermeintlich objektiver Betrachtung wider. Ohne interdisziplinäre, theoretische Arbeit kann diese die beobachteten Phänomene jedoch nur so wiedergeben, wie diese sich selbst darstellen, was auf eine unkritische, affirmative Haltung hinausläuft. Was in der Beziehung mit Menschen recht selbstverständlich ist, nämlich dass wir uns in ihrer Beurteilung nicht auf ihre regelmäßig geschönte - Selbstdarstellung verlassen können, ist in der Analyse des Rechts in den meisten Ländern selten.

Es ist von daher auch nicht verwunderlich, dass die in Frankreich auf der politischen Ebene immerhin erwünschten interdisziplinären, sprich sozialwissenschaftlichen Inhalte und Ansätze, wie sie in der Benennung zahlreicher der o. g. Fächer zum Ausdruck kommen (z. B. Verfassungsrecht und politische Institutionen, Recht und Steuern der Unternehmen, u. a.), ${ }^{49}$ in der Lehrpraxis im besten Fall rein beschreibend, häufig aber auch stark verrechtlicht entwickelt werden. Immerhin wird die Ignoranz der außerrechtlichen Realität in Frankreich nicht so weit getrieben, dass - wie bisweilen etwa in Deutschland - die juristische Arbeit als geisteswissenschaftliche Tätigkeit verstanden

46 Hierzu und zum folgenden im Hinblick vor allem auf Frankreich vgl. P. Legendre (Fn. 3), S. I 54.

47 Vgl. Duncan Kennedy, "Legal Education as Training for Hierarchy«, in: David Kairys (Hrsg.) (Fn. 22),

S. 54-75 sowie ausführlich ders., Legal Education and the Reproduction of Hierarchy. A Polemic Against the System, Afar: Cambridge/Mass. I983.

$48 \mathrm{Zu}$ diesem Absatz vgl. P. Legendre (Fn. 3), S. I 53 ff.

49 Vgl. dazu in historischer Perspektive J. Gatti-Montain (Fn. I9), S. I Iff., I 29 f. 
würde.Jede sog. Geisteswissenschaft ist ja schon allein deshalb Sozialwissenschaft, weil Sprache aus gesellschaftlicher Praxis erwächst. ${ }^{\circ}$ Genährt von der Würdigung staatlicher Autorität herrscht dafür in Frankreich eine Art Staatspositivismus. ${ }^{\text {sI }}$ Dieser führt - in Verbindung mit der auch in Frankreich häufigen Geringschätzung der als nicht exakt geltenden Sozialwissenschaften (obwohl auch Mathematik und Naturwissenschaften nicht exakt und objektiv sein können, sondern historisch und gesellschaftlich determiniert sind) ${ }^{\varsigma_{2}}-$ zu erheblichem Widerstand gegen das Nachdenkenüber Recht.

Trifft die hier entwickelte Erklärung zu, kann die bescheidene Präsenz von Rechtswissenschaft im Jurastudium kaum sinnvoll beklagt werden.53 Wohl aber lässt sich eine juristische Ausbildung im Hinblick darauf kritisieren, inwiefern sie das erstgenannte Ziel, nämlich praxistaugliche Juristen auszubilden, verwirklicht. Das führt uns zu den Arbeitsmethoden in der französischen Juristenausbildung.

Wie auch in anderen Ländern gibt es an den französischen Universitäten und juristischen Ausbildungsstätten neben Vorlesungen auch Travaux dirigés, d. h. Arbeiten unter Anleitung (Übungen). In Jura müssen bis zum DEUG 20\%, im Studienjahr der Licence dann I $5 \%$ aller Lehrveranstaltungen Übungen sein. Wie die französische Benennung schon andeutet, dienen sie der praktischen Erarbeitung und Einübung des in den Vorlesungen vermittelten Stoffes. Doch die vorherrschenden, professoren- und wissenszentrierten Unterrichtsformen bringen es mit sich, dass die Übungen meist gleichfalls wie Vorlesungen ablaufen: Der Übungsleiter, meist ein Doktorand, trägt vor und die Studenten schreiben mit. Deren Beteiligung ist also selbst in dem dafür ausdrücklich vorgesehenen Rahmen selten. Sie ist umso dürftiger, als die Besetzung der Übungsgruppen - an der Universität Metz aus finanziellen Gründen teilweise mit 45 Studenten pro Gruppe - studentische Beteiligung oder gar Diskussion nicht immer zulässt. Aber die Mitarbeit der Studenten ist wohl auch nicht unbedingt erwünscht, wie sich daran ablesen lässt, dass in den Übungsräumen Tische und Stühle kommunikationsfeindlich hintereinander angeordnet sind.

Mehr noch als auch in anderen Ländern sind pädagogische und didaktische Anstrengungen in Frankreich marginal, teilweise sogar verpönt. Durch die Schule bereits gründlich konditioniert, sind die Studenten selbst die ersten, die in Vorlesung wie Übung nach einem Diktat verlangen. Von der fixen Idee geplagt, nicht genug zu wissen, sind sie meist unfähig, spontan einen Standpunkt zu vertreten. Dass sich Kenntnisse und Ideen häufig erst beim Sprechen entfalten, weil >nicht wir wissen, es ist allererst ein gewisser Zustand unsrer, welcher weiß «s4 ist ihnen wie auch vielen Hochschullehrern in keiner Weise bewusst. Im Übrigen betrachten sie ihre Lehrer, einschließlich der häufig nur ein paar Jahre älteren Doktoranden, wie Halbgötter. Die Professoren ihrerseits verachten die Studenten nicht selten. Charakteristisch dafür ist der teilweise auch von ihnen verwendete studentische Ausdruck Colle zur Bezeichnung einer Prüfung. Das normalerweise »Klebstoff« meinende Wort unterstellt, die Rolle einer Prüfung bestehe darin, jemanden durchfallen zu lassen.

50 Vgl. Ludwig Wittgenstein, »Philosophische Untersuchungen«, in: Schriften, Frankfurt/M. I984, Bd. I, S. 279-544 (I. Teil, $\mathbb{S}$ 202-208, 256 ff., grundlegend für das sog., bereits auf K. Marx zurückgehende sog. Privatsprachenargument); ders., »Das Blaue Buch«, ebd., Bd.V, S. ऽr f., ders., »Bemerkungen über die Grundlagen der Mathematik«, ebd., Bd. VI, Teil VI, $\$ 4$ I.

5 I Auf der Grundlage von Auguste Comte, Cours de philosophie positive (I830-I842), ire et $2^{e}$ leçon, Larousse: Paris 1936 u. Raymond Carré de Malberg, Contribution à la théorie générale de l'Etat (1920), 2 Bd., Dalloz: Paris 2003.

52 Vgl. beispielhaft Stephen Jay Gould, The mismeasure of man, Norton \& Co.: New York/London I98 I (Der falsch vermessene Mensch, Frankfurt/M. I988).

53 Eine solche Kritik und die Analyse der gesellschaftlichen und politischen Rolle der Juristenausbildung stehen im Mittelpunkt bei J. Gatti-Montain (Fn. 19).

54 So bereits Heinrich von Kleist, »Über die allmähliche Verfertigung der Gedanken beim Reden«, in: ders., Werke, Bd. 3, Köln 1996, S. 310-3 I6 (3 I 5). 
Es gibt zwei Hauptarten von Prüfungsaufgaben, nämlich Themenarbeiten und Fall-

lösungen. Im Privatrecht kommen beide etwa gleichgewichtig zum Zuge, während im öffentlichen Recht Themenarbeiten überwiegen.ss In den spezialisierten Ausbildungsstätten spielen praxisnähere Aufgaben, insbesondere Falllösungen verständlicherweise eine größere Rolle als theoretische Fragestellungen.

Unter den Themenarbeiten am häufigsten ist die Dissertation. Es handelt sich um ein Essay mit strengen formalen Anforderungen, insbesondere im Hinblick auf die Gliederung (den Plan). Ein ungeschriebenes Gesetz besagt nämlich, dass die Abhandlung aus zwei Hauptteilen mit vorzugsweise jeweils zwei Unterteilen bestehen muss, eingerahmt durch Einleitung und ggf. Schluss. Zwar lässt sich dieses Gliederungsmodell damit rechtfertigen, dass es für Studenten leichter durchführbar ist als eine drei- oder gar vierteilige Struktur, aber seine Verabsolutierung erzieht nicht gerade zum souveränen Umgang mit Darstellungsformen. Wohl noch problematischer ist, dass die in diesem Rahmen gestellten Prüfungsthemen (z. B. >Das französische Parlament seit 1946<, >Die Verantwortlichkeit der Exekutive in der V. Republi$\mathrm{k}^{{ }^{6}}$ ) von den Studenten vor allem verlangen, gewisse Aspekte der zu diesem Zweck auswendiggelernten Vorlesung nachzubeten.

Etwas mehr Anwendung des Erlernten ist im sog. Kommentar gefragt. Dieser gilt deshalb auch als sehr viel schwieriger, zumal er gleichfalls mit Hilfe eines Plan zu strukturieren ist. Beim sog. Textkommentar müssen die Studenten meist die Äußerung einer-mehr oder minder bekannten - Persönlichkeit oder eines juristischen Autoren besprechen. Der Urteilskommentar bezieht sich hingegen auf eine Gerichtsentscheidung und nähert sich der Falllösung an.

Die Falllösung ihrerseits unterliegt weniger strengen formalen Anforderungen als etwa in Deutschland, wahrscheinlich unter dem Einfluss des literarisch geprägten Essays. Teilweise verbirgt sich hinter einer Falllösung gar ein bloßer Katalog von Fragen $\mathrm{zu}$ einem konkreten Fall. ${ }^{77}$ Das folgt wohl auch aus der geringeren Verrechtlichung der französischen Gesellschaft; in Frankreich können die meisten Juristen - auch Richter - mit der formalen Logik, dem Syllogismus und dem »logisch-deduktiven Subsumtionsmodell« nicht viel anfangen. Einerseits mindert das ihre Fähigkeit zur - und die Effektivität der - juristischen Argumentation. Andererseits sollten wir nicht vergessen, dass die formale Logik häufig auch ein Mittel der Manipulation ist, weil sie dazu verführt, die ihr zugrundeliegenden Axiome und Prämissen, ganz zu schweigen von individuellen und gesellschaftlichen Interessen auszublenden..$^{8}$ Insofern hat ihre begrenzte Rolle in Frankreich den positiven Effekt, soziale und politische Fragen nicht in übergroßem Maße einer nur scheinbar objektiven und neutralen, dafür aber entpolitisierenden und entfremdenden Behandlung zu unterwerfen.

Die Methodenfrage ist auch für eine rechtswissenschaftliche Orientierung von erheblicher Bedeutung. Die Falllösung erscheint als Rahmen nämlich ungeeignet, rechtliche Phänomene kritisch zu reflektieren und zu erklären. Dazu dürfte die Form des Essays viel besser passen, auch wenn dieses Potential in Frankreich wegen Formalismus und unkritischen, rein beschreibenden Tendenzen vielfach ungenutzt bleibt. Die wenigen Hauptteile eines Essays wie vor allem einer Doktorarbeit - auf

5s Eine Auswahl korrigierter verfassungsrechtlicher Prüfungsaufgaben aus I 6 französischen Universitäten findet sich in: Yves Jégouzo (Hrsg.), Annales du droit: Droit constitutionnel et institutions politiques. DEUG de droit, I ère année, session 1999, Dalloz: Paris 1999.

56 Ebd., S. I7 ff.

57 Vgl. z. B. ebd., S.9I ff.

58 Vgl. Dieter Grimm, »Methode als Machtfaktor «, Festschrift Helmut Coing, Bd. I, Frankfurt/M. I982, S. 469-492; Michel Miaille, Une introduction critique au droit, F. Maspero: Paris 1980, S. 201-226; Michel Troper, »Fonction juridictionnelle ou pouvoir judiciaire? «, Pouvoirs Nr. I6, I98 I, S. 5-1 5. 
Französisch Thèse, was die Erwartung einer umfassenden Hypothese zum Ausdruck bringt - sollen eben im Idealfall Erklärungsansätze, genauer gesagt Unterhypothesen widerspiegeln. Das kann kaum funktionieren, wenn (wie in manchen deutschen Dissertationen) die Gliederung mehr als vier oder fünf große Etappen enthält.

Was die Einbeziehung praktischer Erfahrungen in das Jurastudium angeht, sieht die Lage in Frankreich nicht besser als etwa in Deutschland aus. Der erste Kontakt mit der Berufspraxis von Juristen erfolgt im fakultativen Praktikum während des Studienjahres der Maîtrise.

Die Methodendiskussion abschließend, ist noch darauf hinzuweisen, dass der Gang zum Repetitor für französische Jurastudenten unbekannt ist. Demgegenüber bedürfen bekanntlich etwa 90\% der angehenden Juristen in Deutschland kostspieliger privater Nachhilfe. Das lässt vermuten, dass die Juristenausbildung in Frankreich im deutsch-französischen Vergleich nicht so schlecht abschneidet, wie manche kritische Zeile dieses Beitrags vielleicht nahelegt.

\section{Andreas Fischer-Lescano* Weltrecht als Prinzip}

\section{Die Strafanzeige in Deutschland gegen Donald Rumsfeld wegen der Folterungen in Abu Ghraib}

Die These, dass nach dem ir. September 200 I zwischen Europa und den USA kategoriale Differenzen im Umgang mit terroristischen Gefahren zu beobachten seien, beruht im Kern auf der Behauptung, dass der alteuropäische Rechtspazifismus versagt habe. Die globale pax americana setze hingegen hobbesianisch auf militärische Macht, und dies sei die adäquate Form, die weltweite Friedensidee zu realisieren. Im Prinzip ist das kein besonders origineller Gedanke. Robert Kagan, ${ }^{\mathrm{I}}$ dem er dennoch zu einiger Prominenz verholfen hat, ist nur der intellektuelle Trittbrettfahrer einer ganzen Reihe klassischer Theoretiker der >Realpolitik<, die sich darin einig waren, dass Konflikte >letztlich nur politisch und nicht rechtlich entschieden werden könnten. Ironischerweise waren die Vordenker dieser These gestandene Alteuropäer. Carl Schmitt wie auch Hans Morgenthau haben den theoretischen Grundstein dieser Formen der Weltbeschreibung gelegt ${ }^{2}$ und darauf insistiert, dass auf globaler Ebene nicht das Recht, sondern das freie Spiel der etatistischen Kräfte herrsche. ${ }^{3}$

Wenn man nun in dieser Tradition das politische System der Nationalstaaten als zentralen Referenzpunkt dafür versteht, was »Europa « und »Amerika « - was immer diese Simplifizierungen bedeuten mögen - auseinander treibt, wird man allerdings den

* Dr. iur., LL.M. (EUI, Florenz); Mitglied der Forschungsgruppe >Internationale Organisation, Demokratischer Friede und die Herrschaft des Rechts< der Hessischen Stiftung Friedens- und Konfliktforschung (HSFK) und wiss. Mitarbeiter am Institut für Wirtschaftsrecht, J. W. Goethe-Universität, Frankfurt am Main.

I Kagan (2002), S. I $94 \mathrm{ff}$.

2 Schmitt (1932), passim; Morgenthau (I940), S. 260 ff.; Kritik hieran und an neueren interdisziplinären Projekten ( $>$ The New Generation of Interdisciplinary Scholarship $<$ ), die das >Weimar-Argument $<$, die Weimarer Argumentationsmuster, reproduzieren, bei Koskenniemi (2000), S. I7 ff.

3 Hans Morgenthau bspw. ging davon aus, dass in den internationalen Beziehungen »a competitive contest for power will determine the victorious social forces, and the change of the existing legal order will be decided, not through a legal procedure [...] but through a conflagration of conflicting social forces which challenge the legal order as a whole« (Morgenthau (I940), S. $275 \mathrm{f}$. 